\title{
Impact of interface roughness on perpendicular transport and domain formation in superlattices
}

\author{
Andreas Wacker and Antti-Pekka Jauho \\ Mikroelektronik Centret, Danmarks Tekniske Universitet, DK-2800 Lyngby, Denmark
}

(Contribution to ICSMM-9 in Liège, Belgium, July 1996

to appear in Superlattices and Microstructures)

\begin{abstract}
A microscopic calculation of the perpendicular current in doped multiple quantum wells is presented. Interface roughness is shown to affect the resonant transitions as well as to cause a nonresonant background current. The theoretical characteristics exhibit several branches due to the formation of electric field domains in quantitative agreement with experimental data.
\end{abstract}

\section{INTRODUCTION}

The electric transport in semiconductor superlattices is usually dominated by resonances between the localized energy levels inside the wells resulting in peaks in the current-field relation. This may yield complicated current-voltage characteristics exhibiting may branches due to the formation of domains of different electric field inside the sample (see, e.g., [1] and references therein). These experimental features could be qualitatively reproduced by theoretical models combining rate equations for the transport between the wells and Poisson's equation [2,3]. While these approaches assume interwell transition rates which are either fitted or obtained from phenomenological models, we have recently proposed a way to calculate the transport microscopically [4]. We obtained good quantitative agreement with the experimental data of Ref. [5] for highly doped samples, where the scattering from ionized impurities causes a strong broadening of the levels. Here we consider the lower doped sample used in [1,6] consisting of a 40 period GaAs/AlAs superlattice (barrier width $b=4$ $\mathrm{nm}$, well width $w=9 \mathrm{~nm}$, period $d=b+w$, doping $N_{D}=1.5 \times 10^{11} / \mathrm{cm}^{2}$ per well, cross section $A=1.13 \times 10^{-4} \mathrm{~cm}^{2}$ ) and investigate the impact of interface roughness which both contributes to the broadening and causes nonresonant transitions between the wells.

\section{THE BASIC TRANSPORT MODEL}

In the case of weakly coupled quantum wells the appropriate basis set is a product of Wannier functions $\Psi^{\nu}(z-n d)$ of subband $\nu$ localized in well $n$, and plane waves $e^{i \underline{k} \cdot \underline{r}}$. Here the $z$ direction is the growth direction and $\underline{k}, \underline{r}$ are vectors within the $(x, y)$ plane. Restricting ourselves to the lowest two minibands (denoted by $a$ and $b$ ) and coupling between neighbouring wells the Hamiltonian in the presence of an electric field $F$ is given by $\hat{H}=$ $\hat{H}_{0}+\hat{H}_{1}$, where 


$$
\begin{aligned}
& \hat{H}_{0}=\sum_{n, \underline{k}}\left[E_{n}^{a}(\underline{k}) a_{n}^{\dagger}(\underline{k}) a_{n}(\underline{k})+E_{n}^{b}(\underline{k}) b_{n}^{\dagger}(\underline{k}) b_{n}(\underline{k})-e F\left(R_{0}^{a b} a_{n}^{\dagger}(\underline{k}) b_{n}(\underline{k})+R_{0}^{b a} b_{n}^{\dagger}(\underline{k}) a_{n}(\underline{k})\right)\right] \\
& \hat{H}_{1}=\sum_{n, \underline{k}}\left[T_{1}^{a} a_{n+1}^{\dagger}(\underline{k}) a_{n}(\underline{k})+T_{1}^{b} b_{n+1}^{+}(\underline{k}) b_{n}(\underline{k})-e F R_{1}^{a b} a_{n+1}^{\dagger}(\underline{k}) b_{n}(\underline{k})-e F R_{1}^{b a} b_{n+1}^{\dagger}(\underline{k}) a_{n}(\underline{k})\right]
\end{aligned}
$$

with $E_{n}^{\nu}(\underline{k})=E^{\nu}+\hbar^{2} k^{2} /\left(2 m_{w}\right)-e F d n$ ( $m_{w}$ is the effective mass in the well), the couplings $R_{h}^{\nu^{\prime} \nu}=\int d z \Psi^{\nu^{\prime}}(z-h d) z \Psi^{\nu}(z)$, and the miniband width $4\left|T_{1}^{\nu}\right|$ of subband $\nu$. Diagonalizing the Hamiltonian $\hat{H}_{0}$ leads to renormalized coefficients in $\hat{H}_{0}$ and $\hat{H}_{1}$ [7] which we use in the following.

We calculate the Wannier functions in a Kronig-Penney-type model. Following Ref. [8] we model the nonparabolicity by an energy dependent effective mass $m(E)=m_{c}(1+(E-$ $\left.E_{c}\right) / E_{g}$ ), where $m_{c}$ is the effective mass at the conduction band minimum of energy $E_{c}$, and $E_{g}$ is the energy gap. Then the usual connection rules hold for the envelope function provided that the momentum matrix element $P$ between the conduction and valence band states is identical in both materials. We use the values $[8] m_{c}^{\mathrm{GaAs}}=0.067 m_{e}, m_{c}^{\mathrm{AlAs}}=0.15 m_{e}$, $E_{g}^{\mathrm{GaAs}}=1.52 \mathrm{eV}, E_{g}^{\mathrm{AlAs}}=3.13 \mathrm{eV}$, and the conduction band discontinuity $\Delta E_{c}=1.06 \mathrm{eV}$. These parameters yield a relation $E(k)=E_{c}+\hbar^{2} k^{2} /(2 m(E))$ which is in excellent agreement with the band structure of AlAs [9] for the energies of interest. W We obtain the coefficients $E^{a}=47.1 \mathrm{meV}, E^{b}=176.6 \mathrm{meV}, T_{1}^{a}=-0.0201 \mathrm{meV}, T_{1}^{b}=0.0776 \mathrm{meV}, R_{1}^{b a}=2.66 \times 10^{-4} d$, and $R_{0}^{b a}=-0.149 d$.

For small couplings between the wells and fast intersubband relaxation the current from subband $\nu$ in well $n$ to subband $\mu$ in well $n+1$ is given by the following expression [10]:

$I_{n \rightarrow n+1}^{\nu \rightarrow \mu}=2 e \sum_{\underline{\underline{k}^{\prime}, \underline{k}}}\left|H_{(n+1) \underline{k}, \underline{\underline{k}^{\prime}}}^{\mu}\right|^{2} \int_{-\infty}^{\infty} \frac{d \mathcal{E}}{2 \pi \hbar} A_{n}^{\nu}\left(\underline{k}^{\prime}, \mathcal{E}\right) \cdot A_{n+1}^{\mu}(\underline{k}, \mathcal{E}-\Delta \mu)\left[n_{F}(\mathcal{E})-n_{F}(\mathcal{E}-\Delta \mu)\right]$.

Here $A_{n}^{\nu}(\underline{k}, \mathcal{E})$ is the spectral function of subband $\nu$ in well number $n$ and $n_{F}(\mathcal{E})=\left(1+e^{\beta \mathcal{E}}\right)^{-1}$ is the Fermi function. The energy $\mathcal{E}$ is measured with respect to the electrochemical potential $\mu_{n}$ in well $n$ yielding an implicit dependence of $A_{n}^{\nu}(\underline{k}, \mathcal{E})$ on $\mu_{n}$. We determine $\mu_{n}$ from the local electron density $n_{n}=\sum_{\underline{k}} \int_{-\infty}^{\infty} d \mathcal{E} n_{F}(\mathcal{E}) A_{n}^{a}(\underline{k}, \mathcal{E}) /(\pi A)$. Then the difference $\Delta \mu=$ $\mu_{n+1}-\mu_{n}$ is equal to $-e F d$ for $n_{n}=n_{n+1}$. We obtain $A_{n}^{\nu}(\underline{k}, \mathcal{E})$ in equilibrium from the retarded self-energy $\Sigma_{n}^{\nu \text { ret }}(\underline{k}, \mathcal{E})$ neglecting the coupling to the other wells. In Ref. 四 we have calculated the self-energy for scattering from the screened potential of ionized impurities within the self-consistent single-site-approximation. As an additional contribution to the self-energy we study here the impact of interface roughness.

\section{MODELLING OF INTERFACE ROUGHNESS}

We consider an interface located at $z=z_{0}$ exhibiting thickness fluctuations $\xi(\underline{r})$ of the order of $\pm \eta$ (we use $\eta=2.8 \AA$ which is one monolayer of GaAs). We assume $\langle\xi(\underline{r})\rangle_{r}=0$

\footnotetext{
${ }^{1}$ Within this approach $P=\hbar \sqrt{E_{g} /\left(2 m_{c}\right)}$ is slightly different for the two materials in contrast to the assumption. Furthermore, the envelope functions for different energies are not orthogonal as the effective Hamiltonian is energy dependent. However, the overlap is small and we neglect these complications.
} 
and the correlation

$$
\left\langle\xi(\underline{r}) \xi\left(\underline{r}^{\prime}\right)\right\rangle_{r}=\alpha \eta^{2} \exp \left(-\left|\underline{r}-\underline{r}^{\prime}\right| / \lambda\right) .
$$

This can be motivated in the following way: At a given point $\underline{r}$ there is an island of thickness $\eta$ with a probability $\alpha$ (we use an average coverage $\alpha=0.5$ ). Therefore $\langle\xi(\underline{r}) \xi(\underline{r})\rangle_{r}=\alpha \eta^{2}$. Provided the island extends from $\underline{r}$ to $\underline{r}^{\prime}$ we assume a constant probability to find a further neighbouring atom beyond $\underline{r}^{\prime}$ yielding the exponential in Eq. (2). Following Ref. [11] we model the additional potential by a $\delta$-function at the perfect interface $U(\underline{r}, z)=\xi(\underline{r}) \Delta E_{c} \delta\left(z-z_{0}\right)$ and obtain

$$
\begin{aligned}
\hat{H}^{\mathrm{rough}}=\frac{1}{A} \sum_{\underline{k}, \underline{p}, h}[ & U_{h}^{a a}(\underline{p}) a_{n+h}^{\dagger}(\underline{k}+\underline{p}) a_{n}(\underline{k})+U_{h}^{b b}(\underline{p}) b_{n+h}^{\dagger}(\underline{k}+\underline{p}) b_{n}(\underline{k}) \\
& \left.+U_{h}^{b a}(\underline{p}) b_{n+h}^{\dagger}(\underline{k}+\underline{p}) a_{n}(\underline{k})+U_{h}^{a b}(\underline{p}) a_{n+h}^{\dagger}(\underline{k}+\underline{p}) b_{n}(\underline{k})\right]
\end{aligned}
$$

with the matrix elements $U_{h}^{\nu \mu}(\underline{p})=\int d^{2} r e^{-i \underline{p} \cdot \underline{r}} \Delta E_{c}\left[\xi(\underline{r}) \Psi^{\nu *}\left(z_{0}-h d\right) \Psi^{\mu}\left(z_{0}\right)\right]$. The elements $U_{1}^{\nu \mu}$ contribute to the current from one well to the next via Eq. (11). For weakly coupled wells $U_{2}^{\nu \mu}, U_{3}^{\nu \mu}, \ldots$ are small and are neglected in the following. The elements $U_{0}^{\nu \mu}$ result in scattering within the wells. We calculate their contribution to the self-energy within the self-consistent Born-Approximation

$$
\sum_{\text {rough }}^{a \operatorname{ret}}(\underline{k}, \mathcal{E})=\frac{2}{A^{2}} \sum_{\underline{k}^{\prime}} \frac{\left|U_{0}^{a a}\left(\underline{k}-\underline{k}^{\prime}\right)\right|^{2}}{\mathcal{E}+\mathcal{E}_{n}^{F}-E_{k^{\prime}}-\sum_{n}^{a \operatorname{ret}}\left(\underline{k}^{\prime}, \mathcal{E}\right)},
$$

where the factor 2 takes into account the two interfaces per well. The calculation for the subband $b$ is performed in the same way. With (2) we obtain the matrix element

$$
\left|U_{0}^{a a}(\underline{p})\right|^{2}=A \Delta E_{c}^{2}\left|\Psi_{a}\left(z_{0}\right)\right|^{4} \frac{2 \pi \alpha \eta^{2} \lambda^{2}}{\left(1+(p \lambda)^{2}\right)^{3 / 2}}
$$

In contrast to this the usual choice (see, e.g., [11]) $\left\langle\xi(\underline{r}) \xi\left(\underline{r}^{\prime}\right)\right\rangle_{r}=\alpha \eta^{2} \exp \left(-\left|\underline{r}-\underline{r}^{\prime}\right|^{2} / \lambda^{2}\right)$ yields $\left|U_{0}^{a a}(\underline{p})\right|^{2} \sim \exp \left(-\lambda^{2} p^{2} / 4\right)$. In order to compare both expressions we consider the matrix element for scattering at a single circular island of thickness $\eta_{i}$ and radius $\lambda_{i}$ which is given by $U_{0}^{a a i}(\underline{p})=2 \pi \eta_{i} \Delta E_{c}\left|\Psi_{a}\left(z_{0}\right)\right|^{2} \lambda_{i} J_{1}\left(p \lambda_{i}\right) / p$, where $J_{1}$ is the Bessel function of the first kind. Now there are $\alpha A /\left(\pi \lambda^{2}\right)$ islands and we assume that negative and positive $\eta_{i}$ cancel each other. Then $\left|U^{a a}(\underline{p})\right|^{2}=\sum_{i}\left|U_{0}^{a a i}(\underline{p})\right|^{2} \sim p^{-3}$ for large $p$ as in Eq. (5) with a prefactor differing by $2 / \pi$. This indicates that the choice (2) captures an essential part of physics not contained in the usual choice.

Furthermore, the single island result reflects the quality of the Born-approximation for the self-energy. The analysis of a $\delta$-potential reveals that diagrams containing multiple scattering at a single island become important if the product $U(p) m /\left(2 \hbar^{2}\right)$ is larger than 1. Using the value $U_{0}^{a a i}(\underline{0})$ this yields $2 \hbar^{2} /\left(\pi m \lambda^{2}\right)<\eta \Delta E_{c}\left|\Psi_{a}\left(z_{0}\right)\right|^{2}$. (For our parameters $\lambda>15 \mathrm{~nm}$.) This expression can be interpreted easily: The right hand side is the energy the electron gains if it is located at an island exhibiting a larger well width. The left hand side is the quantization energy associated with a length $\lambda$. The localization of the wavefunction takes place if the energy gain due to the larger well width dominates the cost due to the localization. In this case higher order diagrams become important. 


\section{RESULTS AND DISCUSSION}

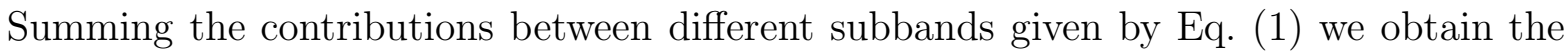
current $I_{n \rightarrow n+1}\left(F, n_{n}, n_{n+1}\right)$ depending on the field and the electron densities. Fig. 1a shows $I_{n \rightarrow n+1}\left(F, N_{D}, N_{D}\right)$ exhibiting a first maximum $I=0.33 \mathrm{~mA}$ at $e F d=10 \mathrm{meV}$ where the current is dominated by $T_{1}^{a}$ transitions and a second peak $I=1.8 \mathrm{~mA}$ at $e F d=140 \mathrm{meV}$ due to the $a \rightarrow b$ resonance. The interface roughness has two implications: Firstly the maxima are broadened and slightly shifted due to the contribution to the self-energies via Eq. (1). Secondly there is a nonresonant current due to the $U_{1}^{a a}$ transitions dominating the behaviour between the maxima.

In order to study the domain formation we consider effective fields $F_{i}$ between wells $i$ and $i+1$ fulfilling the discretized Poisson equation $\epsilon_{r} \epsilon_{0}\left(F_{i}-F_{i-1}\right)=e\left(n_{i}-N_{D}\right)$. Then the

local currents $I_{i \rightarrow i+1}\left(F_{i}, n_{i}, n_{i+1}\right)$ have to be equal for all $i$ in the steady state. The total voltage across the superlattice is $U=d \sum_{i=1}^{N} F_{i}$. Using the boundary conditions $n_{1}=2 N_{D}$, $n_{N+1}=2 N_{D}$ we obtain the current-voltage characteristic shown in Fig. 1b which exhibits the usual sequence of branches [1,2]. In contrast to previous theoretical results the maximal current of the branches $(90 \mu \mathrm{A})$ is significantly lower than the height of the first resonance. It is almost independent of the boundary conditions, which mainly affect the voltage where the leftmost homogeneous branch breaks up. The maximal current of $90 \mu \mathrm{A}$ is between the two experimental results [6] $60 \mu \mathrm{A}$ and $130 \mu \mathrm{A}$ for samples \#1 and \#2, respectively, which both have the nominal sample parameters used here. We can reproduce the difference in current between both samples by assuming barrier widths which differ by just one monolayer, thus suggesting a possible explanation for the experimental discrepancy. The minimal currents of the branches $(26 \mu \mathrm{A})$ seem to be lower than the experimental values. They depend strongly on the nonresonant currents, e.g., we obtain the value $10 \mu \mathrm{A}$ ignoring transitions via $U_{1}$. This indicates that we have probably underestimated the nonresonant current. The slope of the branches is steeper in our calculation than found experimentally; this could be due to some additional contact resistance of the order of $1 \mathrm{k} \Omega$ which is not included in our calculation.

In conclusion, we have presented a microscopic model for sequential tunnelling in weakly coupled quantum wells. The model describes the domain formation in superlattices quantitatively with no fitting parameters. Nonresonant transitions due to interface roughness have been shown to strongly affect the current between the resonances and the extent of the current branches. 


\section{REFERENCES}

[1] S. H. Kwok, H. T. Grahn, M. Ramsteiner, K. Ploog, F. Prengel, A. Wacker, E. Schöll, S. Murugkar, and R. Merlin, Phys. Rev. B 51, 9943 (1995).

[2] F. Prengel, A. Wacker, and E. Schöll, Phys. Rev. B 50, 1705 (1994), erratum in 52, 11518 (1995).

[3] L. L. Bonilla, J. Galán, J. A. Cuesta, F. C. Martínez, and J. M. Molera, Phys. Rev. B 50, 8644 (1994).

[4] A. Wacker and A.-P. Jauho, Physica Scripta, in print.

[5] P. Helgesen, T. G. Finstad, and K. Johannessen, J. Appl. Phys. 69, 2689 (1991).

[6] G. Schwarz, A. Wacker, F. Prengel, E. Schöll, J. Kastrup, H. T. Grahn, and K. Ploog, Semicond. Sci. Technol. 11, 475 (1996).

[7] R. F. Kazarinov and R. A. Suris, Sov. Phys. Semicond. 6, 120 (1972).

[8] G. Brozak, E. A. de Andrada e Silva, L. J. Sham, F. DeRosa, P. Miceli, S. A. Schwarz, J. P. Harbison, L. T. Florez, and J. S. Allen, Phys. Rev. Lett. 64, 471 (1990).

[9] J. N. Schulman and Y.-C. Chang, Phys. Rev. B 31, 2056 (1985).

[10] G. D. Mahan, Many-Particle Physics (Plenum, New York, 1990).

[11] I. Dharssi and P. N. Butcher, J. Phys.: Condens. Matter 2, 4629 (1990). 


\section{FIGURES}
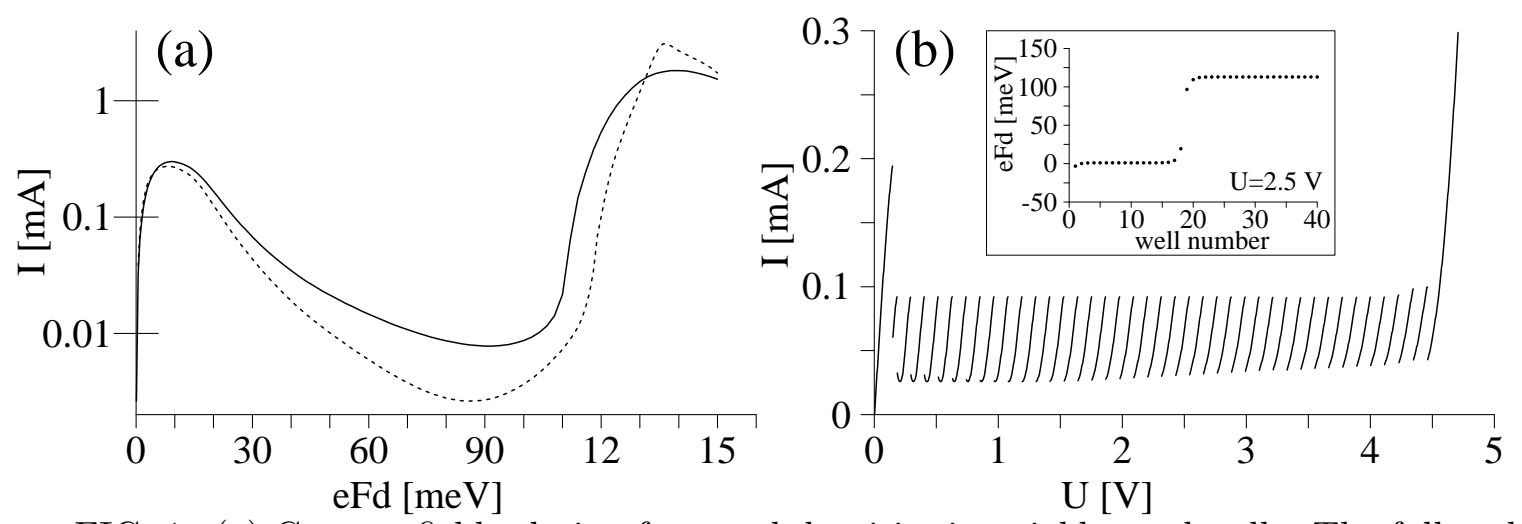

FIG. 1. (a) Current-field relation for equal densities in neighboured wells. The full and dotted lines are calculated with and without interface roughness, respectively (assuming $\lambda=7 \mathrm{~nm}$ ). (b) Current-voltage characteristics for a superlattice consisting of $N=40$ wells for voltage sweep-up. The inset gives the field profile at $U=2.5 \mathrm{~V}$. 